\title{
Vibration of Simply Supported Compound Beams to Moving Loads
}

Jong-Dar Yau

Department of Architecture and Building Technology, Tamkang University, Taipei, Taiwan 10620, R.O.C., jdyau@mail.tku.edu.tw

Follow this and additional works at: https://jmstt.ntou.edu.tw/journal

Part of the Civil and Environmental Engineering Commons

\section{Recommended Citation}

Yau, Jong-Dar (2004) "Vibration of Simply Supported Compound Beams to Moving Loads," Journal of Marine Science and Technology. Vol. 12: Iss. 4, Article 11.

DOI: $10.51400 / 2709-6998.2252$

Available at: https://jmstt.ntou.edu.tw/journal/vol12/iss4/11

This Research Article is brought to you for free and open access by Journal of Marine Science and Technology. It has been accepted for inclusion in Journal of Marine Science and Technology by an authorized editor of Journal of Marine Science and Technology. 


\section{Vibration of Simply Supported Compound Beams to Moving Loads}

\section{Acknowledgements}

The research reported herein has been sponsored in part by the National Science Council of the Republic of China through grants No. NSC93-2211-E-032-010. Such a financial aid is gratefully acknowledged. Parts of the illustrative examples of this paper is modified from the paper entitled "Non-linear Vibrations of Simple Truss-Beams to Moving Loads," presented at the Book of Abstract, 5th Euromech Solid Mechanics Conference ESMC003 [25], held during Aug., 2003 at Thessaloniki, Greece. 


\title{
VIBRATION OF SIMPLY SUPPORTED COMPOUND BEAMS TO MOVING LOADS
}

\author{
Jong-Dar Yau
}

Key words: nonlinear vibration, moving load, resonance, speed parameter.

\begin{abstract}
This paper is aimed at investigating the dynamic response of a compound beam subjected to moving loads at high speeds. The compound beam structure is composed of a simple beam and a twomember truss connected by a set of rigid bars at the midpoint of the beam. Based on the nonlinear elastic theory of a symmetric twomember truss under the action of a vertical tip load, and the incremental-iterative solution procedures for solving a nonlinear dynamic system, the dynamic response of the compound beam structure under the excitation of moving loads is studied. According to the numerical results, the nonlinear effect of vibration is not so obvious to the response of the beam but the installation of a two-member truss system in a simple beam may significantly results in smaller dynamic response of the compound beam structure under the passage of moving loads at high speeds.
\end{abstract}

\section{INTRODUCTION}

The dynamic response of beam-like structures caused by a series of moving loads is an important consideration in the vibration analysis of elevated bridges to high-speed trains. By modeling a bridge as a beamlike structure and a vehicle as a moving load, moving mass or moving sprung mass, numerous researchers [3, $4,18,21,22]$ have analytically investigated the moving-vehicle induced vibration of the bridge. In the books written by Fryba [3, 4], the author proposed many theoretical models to study the dynamic problems of railway bridges subjected to moving vehicles. Recently, Yang et al. [22] derived a closed form solution for the dynamic response of simple beams subjected to a series of moving loads. In their studies, the phenomena of resonance and cancellation of railway bridges caused by moving trains and the optimized criteria of determining the span length for high-speed railway bridges have

Paper Submitted 04/30/04, Accepted 10/14/04. Author for Correspondence: Jong-DarYau.E-mail: jdyau@mail.tku.edu.tw.

*Department of Architecture and Building Technology, Tamkang University, Taipei, Taiwan 10620, R.O.C. been carried out. Considering the insertion of elastic bearing into bridges, Yau et al. [26] analytically presented a closed form solution to explain the mechanism of amplification for the train-induced resonance response of an elastically supported beam. Such a resonant phenomenon was also verified by experiments in reference [19]. Moreover, Yau [24] performed the finite element analysis to explore the effect of multiple resonant peaks of continuous bridges subjected to high speed trains. The numerical results show that the coincidence of some of the excitation frequencies implied by the wheel loads moving at different speeds with any of the natural frequencies of continuous bridges may result in the resonance response of continuous railway bridges.

For the sake of increasing the structural safety of high speed railway bridges and the riding comfort of passing trains, especially, the resonant conditions mentioned above is considered, it is necessary to mitigate the dynamic responses of the bridges and vehicles through strengthening the structural stiffness or using vibration absorbers $[9,15,27]$. For the vibration reduction of a railway bridge due to moving trains, one of the effective mechanisms for stiffness strengthening is to install a truss system in the bridge so that the response of the strengthened bridge can be mitigated below the tolerance limit under the passage of high speed trains. The main purpose of this paper is to investigate the strengthening effect of attaching a two-member truss to a simple beam and then to study the dynamic response of such a compound beam structure under the excitation of moving train loads at high speeds. Based on the nonlinear elastic theory of a symmetric two-member truss subjected to a vertical tip load [20] and the incremental-iterative procedures of solving a nonlinear dynamic system [16, 23], the dynamic response of the compound beam caused by a series of moving loads with identical weight and constant interval is studied. According to the numerical results, increasing the aspect ratio of the height of a two-member truss with respect to the beam span can result in smaller resonant 
response of a compound beam than that of a simple beam.

\section{FORMULATION}

Figure 1 shows a sequence of moving loads with identical interval $d$ and constant speed $v$ along the centerline of a simple beam with a two-member truss connected by rigid bars. To simplify the formulation of theory, the rigid bar is modeled as a two-force member and its inclination with respect to the perpendicular line of the beam axis is assumed to be very small so that the transmission of the horizontal force components from the rigid bar to the main beam can be neglected, as depicted in Figure 2. Considering the nonlinear effect of an elastic beam [2, 13, 14], the equations of motion for a Bernoulli-Euler beam traveled by a series of moving loads with constant speed $v$ and identical interval $d$ are written as

$$
\begin{aligned}
& m \ddot{u}-E A\left(u^{\prime \prime}+w^{\prime} w^{\prime \prime}\right)=0 \\
& \left.m \ddot{w}+c \dot{w}+E I w^{<4>}-E A\left[w^{\prime} u^{\prime}+\left(w^{\prime}\right)^{3} / 2\right)\right]^{\prime} \\
& +F_{y} \delta(x-L / 2)=p(x, t)
\end{aligned}
$$

where $m=$ mass per unit length of the beam, $u(x, t)=$ axial displacement of the beam, $w(x, t)=$ transverse deflection of the beam, $c=$ damping coefficient of the beam, $E A=$ axial rigidity, $E I=$ flexural rigidity of the beam, $x=$ beam axis, $L=$ span length, $h=$ vertical projection length of the rigid bar, and $F_{y}=$ vertical reaction transferred from the rigid bar supported by a two-member truss system. The notations ' $\bullet$ and "' represent partial derivative of time $t$ and length $x$, respectively. The load function $p(x, t)$, which is used to describe the moving effect of a series of moving loads acting on the position $x$ of the main beam at time $t$, is expressed as $[22,26]$

$$
\begin{aligned}
p(x, t) & =P \sum_{k=1}^{N} \delta\left[x-v\left(t-t_{k}\right)\right] \times\left[H\left(t-t_{k}\right)\right. \\
& \left.-H\left(t-t_{k}-L / v\right)\right]
\end{aligned}
$$

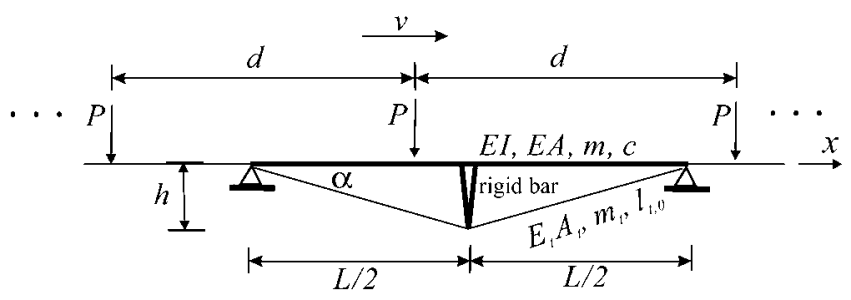

Fig. 1. Simply supported compound beam subjected to uniform moving loads. where $\delta=$ Dirac's delta function, $H(t)=$ unit step function, $N=$ total number of moving loads, and $t_{k}=(k$ $-1) d / v=$ arriving time of the kth load on the beam. And the boundary conditions of both ends for the compound beam are

$$
\begin{aligned}
& u(0, t)=u(L, t)=0 \\
& w(0, t)=w(L, t)=0 \\
& E I w^{\prime \prime}(0, t)=E I w^{\prime \prime}(L, t)=0
\end{aligned}
$$

For the deformed truss members of the compound beam shown in Figure 2, by considering a half portion of the symmetric two-member truss subjected to the action of a half of the vertical tip load, the joint displacement and force equilibrium can be described in Figure 3. According to Hook's law for an elastic truss member, the axial strain ex and the internal forces $f_{x}$ in the truss member caused by the half vertical reaction force $F_{y} / 2$ acting at the tip node are given by [17]

$$
\begin{aligned}
& e_{x}=\frac{l_{t, 2}^{2}-l_{t, 0}^{2}}{2 l_{t, 0}^{2}}=\left(\frac{\Delta_{0}}{l_{t, 0}^{2}}\right)\left(h+\frac{\Delta_{0}}{2}\right) \\
& f_{x}=E_{t} A_{t} e_{x} \frac{l_{t, 2}}{l_{t, 0}}
\end{aligned}
$$

where $h=$ the height of the two-member truss, $\Delta_{0}=$ midpoint deflection of the beam, $l_{t, 0}=$ undeformed length of the truss member, and $E_{t} A_{t}=$ axial rigidity of truss member, $l_{t, 2}=$ deformed length of the truss member, and

$$
\begin{aligned}
& l_{t, 0}=\sqrt{(L / 2)^{2}+h^{2}} \\
& l_{t, 2}=\sqrt{(L / 2)^{2}+\left(h+\Delta_{0}\right)^{2}}
\end{aligned}
$$

Based on the force equilibrium in the vertical direction shown in Figure 3, the vertical component $R_{y}$ of the internal force $f_{x}$ in the truss member can be expressed in terms of $\Delta_{0}$ as [17]

$$
R_{y}=f_{x} \frac{h+\Delta_{0}}{l_{t, 2}}=\frac{E_{t} A_{t} e_{x}}{l_{t, 0}}\left(h+\Delta_{0}\right)
$$

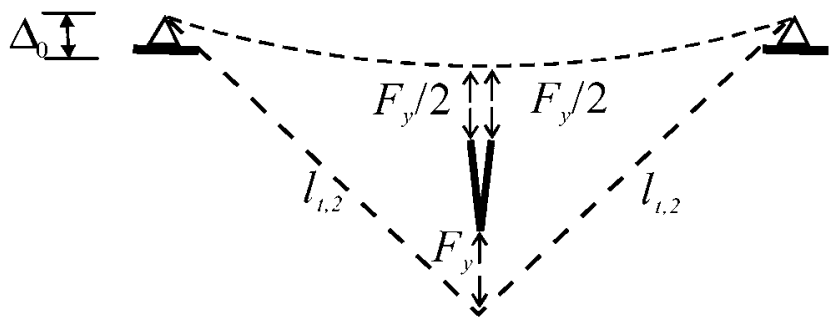

Fig. 2. Deformed configuration. 
Substituting Equations (4) and (6b) into Equation (6a) yields

$$
R_{y}=\frac{E_{t} A_{t}}{2 l_{t, 0}^{3}} \Delta_{0}\left(h+\Delta_{0}\right)\left(2 h+\Delta_{0}\right)
$$

With the concept of lumped mass for the two-member truss and the rigid bars attached to the tip node of the truss, the reaction force $F_{y}$ transferred from the twomember truss system to the midpoint of the main beam is written as [20]:

$$
\begin{aligned}
& F_{y}=M \ddot{\Delta}_{0}+c_{t} \dot{\Delta}_{0}+\frac{E_{t} A_{t}}{l_{t, 0}^{3}} \Delta_{0}\left(h+\Delta_{0}\right)\left(2 h+\Delta_{0}\right) \\
& M=m_{t} l_{t, 0}+m_{r} h
\end{aligned}
$$

where the subscript ' $t$ ' represents the truss and $m_{t}=$ the mass per unit length, $E_{t} A_{t}=$ axial rigidity, $c_{t}=$ damping coefficient, and $m_{r}=$ the mass per unit length of the rigid bar.

In general, the transverse vibration of a simple beam caused by moving loads, as depicted in Figure 1, is mainly concerned in practice. Therfore, Equation (1) can be reduced to a single differential equation by neglecting the inertial effect in the longitudinal direction of the main beam. This leads Equation (1a) to

$$
E A\left(u^{\prime \prime}+w^{\prime} w^{\prime \prime}\right)=0
$$

Integrating Equation (9) twice with respect to $x$ and taking into account the boundary conditions shown in Equation (3a), one can derive the following relations:

$$
\begin{aligned}
& u=-\frac{1}{2} \int_{0}^{x}\left(w^{\prime}\right)^{2} d x+\frac{x}{2 L} \int_{0}^{L}\left(w^{\prime}\right)^{2} d x \\
& u^{\prime}=-\frac{\left(w^{\prime}\right)^{2}}{2}+\frac{1}{2 L} \int_{0}^{L}\left(w^{\prime}\right)^{2} d x
\end{aligned}
$$

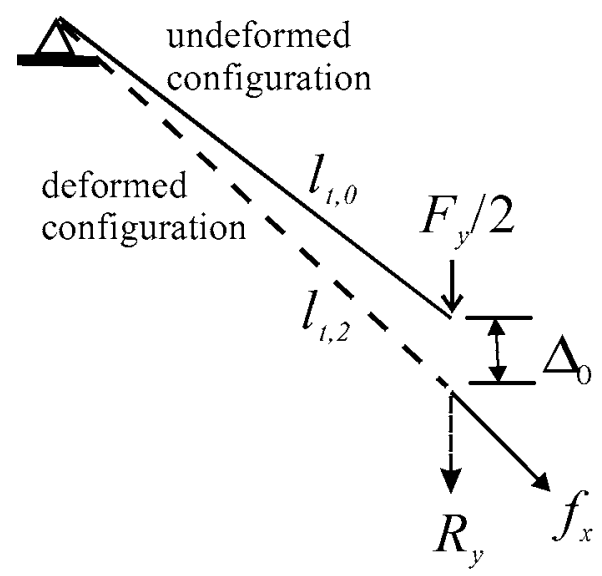

Fig. 3. Joint displacement and force equilibrium.
The substitution of the expression of axial displacement in Equation (10) and the reaction force $F_{y}$ in Equaiton (8) into Equation ( $1 \mathrm{~b}$ ) yields

$$
\begin{aligned}
& m \ddot{w}+c \dot{w}+E I w^{<4>}-\frac{E A}{2 L} w^{\prime \prime} \int_{0}^{L}\left(w^{\prime}\right)^{2} d x \\
& +\left[M \ddot{\Delta}_{0}+c_{t} \dot{\Delta}_{0}+\frac{E_{t} A_{t}}{l_{t, 0}^{3}} \Delta_{0}\left(h+\Delta_{0}\right)\left(2 h+\Delta_{0}\right)\right] \delta(x-L / 2) \\
& =p(x, t)
\end{aligned}
$$

Equation (11) describes the nonlinear transverse vibration of a simple beam installed with a two-member truss system to moving loads. It is noted that there exist highly nonlinear terms related to the midpoint deflection $\Delta_{0}$ of the main beam, which is in general relevant to the maximum displacement and bending moment responses of a simple beam. On the other hand, the forth term in Eq. (11), i.e., $E A / 2 L \times\left[w^{\prime \prime} \int_{0}^{L}\left(w^{\prime}\right)^{2} d x\right]$, is a nonlinearly high order term, its influence to the response of the beam system might not be so obvious in comparison with that of the installation of two-truss members. Without loss of generality, the contribution of such a high order term is also taken into account in the dynamic analysis of the beam system subjected to sequential moving loads in this study.

\section{VIBRATION ANALYSIS}

To solve the equation of motion in Equation (10), the virtual work method is suggested in this study. Based on the boundary conditions of simple supports shown in Equations (3b) and (3c), one can use the function $\sin (n \pi x / L)$ to represent the deformed shape of the main beam. Therefore, the displacement $w(x, t)$ and midpoint deflection $\Delta_{0}[=w(L / 2, t)]$ of the main beam and the integration term in Equation (11) can be represented in a series form as

$$
\begin{aligned}
& w(x, t)=\sum_{n=1}^{\infty} q_{n}(t) \sin \frac{n \pi x}{L} \\
& \Delta_{0}=w\left(\frac{L}{2}, t\right)=\sum_{n=1}^{\infty} q_{n}(t) \sin \frac{n \pi x}{L} \\
& \int_{0}^{L}\left(w^{\prime}\right)^{2} d x=\frac{L}{2} \sum_{j=1}^{\infty}\left(\frac{j \pi}{L} q_{j}(t)\right)^{2}
\end{aligned}
$$

where $q_{n}(t)$ denotes the generalized coordinate associated with the nth vibration shape function $\sin (n \pi x / L)$. By substituting the preceding expression of deformation $w(x, t)$ in Equation (12a) into Equation (11), multiplying both sides of Equation (1) by the shape function 
$\sin (n \pi x / L)$, and then integrating with respect to the beam length $L$, one can derive the generalized equation of motion for the beam with two-member trusses as

$$
\ddot{q}_{2}+2 \xi_{n} \omega_{n} \dot{q}_{n}+\omega_{n}^{2} q_{n}+\bar{F}_{n}(t), \quad n=1,2,3 \ldots
$$

and

$$
\begin{aligned}
& \omega_{n}=\left(\frac{n \pi}{L}\right)^{2} \sqrt{\frac{E I}{m}} \\
& \xi_{n}=\frac{c}{2 m \omega_{n}} \\
& F_{n}(t)=\frac{2 P}{m L} \sum_{k=1}^{N}\left(\sin \frac{n \pi v\left(t-t_{k}\right)}{L}\right. \\
& \left.\times\left[H\left(t-t_{k}\right)-(-1)^{n} H\left(t-t_{k}-\frac{L}{v}\right)\right]\right) \\
& \bar{F}_{n}(t)=\frac{2 F_{y}}{m L} \sin \frac{n \pi}{2}+\frac{E A}{m}\left(\frac{n \pi}{L}\right)^{2}\left[\sum_{j=1}^{\infty}\left(\frac{j \pi}{L} q_{j}\right)^{2}\right] q_{n}
\end{aligned}
$$

where $\omega_{n}=$ the nth frequency of a simple beam, $\xi_{n}=$ damping ratio, and $F_{n}(t)=$ generalized forcing function for representing the moving effect of the train loads over the main beam of the vibrating system. Equation (14d) shows that the internal force term $\bar{F}_{n}(t)$ gives a stiffness strengthening effect to the main beam structure through the installation of a two-member truss system. On account of the strengthening effect of attaching a two-member truss to the beam structure, the dynamic response of the compound beam induced by the train-loads moving at resonant speeds mentioned in reference $[22,26]$ will be implemented in the following section.

\section{PHENOMENON OF RESONANCE}

To clearly demonstrate the resonance phenomenon of a simply supported compound beam subjected to moving train-loads, let us neglect the damping effect of the beam with two-member trusses and the nonlinear terms of the midpoint deflection such as $\Delta_{0}^{2}$ and $\Delta_{0}^{3}$ in Equations (13) and (14), then the nonlinear differential equation of motion in Equation (13) is reduced to:

$$
\begin{aligned}
& \ddot{q}_{n}+\omega_{n}^{2} q_{n}+\frac{2}{m L}\left[M \ddot{\Delta}_{0}+c_{t} \dot{\Delta}_{0}+\frac{2 E_{t} A_{t} h^{2}}{l_{t, 0}^{3}} \Delta_{0}\right] \sin \frac{n \pi}{2} \\
& =F_{n}(t)
\end{aligned}
$$

Moreover, let us consider only the midpoint deflection response of the compound beam, Equation (15) can be further approximately expressed in terms of the first generalized coordinate $q_{1}$ as:

$$
\begin{aligned}
& \left(1+\frac{2 M}{m L}\right) \ddot{q}_{1}+\left(\omega_{1}^{2}+\frac{4 E_{t} A_{t} h^{2}}{m L l_{t, 0}^{3}}\right) q_{1} \\
& =\frac{2 P}{m L} \sum_{k=1}^{N}\left(\sin \frac{\pi v\left(t-t_{k}\right)}{L} \times\left[H\left(t-t_{k}\right)+H\left(t-t_{k}-\frac{L}{v}\right)\right]\right)
\end{aligned}
$$

By following the same analytical procedure for finding the dynamic response of a simple beam traveled by the moving train-loads with equidistant intervals in reference [22], one can derive a closed form solution of Equation (16) in the form:

$$
\begin{aligned}
q_{1} & =\Delta_{s t} \times\left[Q_{1}(v, t) H\left(t-t_{N}\right)\right. \\
& \left.+Q_{2}(\mathrm{v}, \mathrm{t}) H\left(t-t_{N}-L / v\right)\right]
\end{aligned}
$$

$$
\begin{aligned}
& \Delta_{s t}=\frac{2 P L^{3}}{\pi^{4} E I}\left[1+\frac{4 E_{t} A_{t} L^{2}(\sin \alpha \times \sin 2 \alpha)}{\pi^{4} E I}\right]^{-1} \\
& Q_{1}(v, t)=\frac{\sin \Omega\left(t-t_{N}\right)-\bar{S} \sin \omega_{0}\left(t-t_{N}\right)}{1-\bar{S}^{2}} \\
& Q_{2}(v, t)=-\frac{2 \bar{S} \cos \left(\frac{\omega_{0} L}{2 v}\right)}{1-\bar{S}^{2}} \times\left[\sin \omega_{0}\left(t-\frac{L}{2 v}\right)\right. \\
& \left.+\sin \omega_{0}\left(t-\frac{t_{N}+L / v}{2}\right) \times \frac{\sin \left[(N-2) \omega_{0} d / 2 v\right.}{\sin \left(\omega_{0} d / 2 v\right)}\right]
\end{aligned}
$$

$$
\omega_{0}=\omega_{1} \sqrt{\frac{1+4 E_{t} A_{t} L^{2}(\sin \alpha \times \sin 2 \alpha) /\left(\pi^{4} E I\right)}{1+2 M / m L}}
$$

$(17 a-e)$

where $\Delta_{s t}=$ the approximate static deflection of a simple beams with two-member trusses under the action of a concentrated force $P$ at the midpoint of the main beam, $\omega_{0}=$ the fundamental frequency, $\alpha=$ the angle between the main beam and the inclined truss member, that is, $\tan \alpha=2 h / L$, and $\bar{S}=$ the speed parameter that represents the ratio of the driving frequency $\Omega(=\pi v / L)$ to the fundamental frequency $\omega_{0}$ shown in Equation (17e), that is,

$$
\bar{S}=\Omega / \omega_{0}=\pi v /\left(\omega_{0} L\right)
$$


Equation (17e) shows that the expression of the fundamental frequency is dependent upon the rigidity ratio $E_{t} A_{t} L^{2} / E I$, the aspect ratio $(h / L)$ and the mass ratio $M / m L$.

According to the displacement response in Equation (17), it is seen that the term $Q_{1}(v, t)$ is associated with $H\left(t-t_{N}\right)$, which represents the dynamic response induced by the Nth moving load acting on the beam, and the term $Q_{2}(v, t)$ is associated with $H\left(t-t_{N-1}-L / v\right)$, which represents the residual response induced by the $N-1$ moving loads that have passed through the beam. Meanwhile, for the special case with $M=0$ and $\alpha=0$ in Equation (17), which implies that the response of the compound beam reduces to that of the corresponding simple beams [22].

On the other hand, the mathematical expression of the dynamic factor $Q_{2}(v, t)$ shown in Equation (17d) will become an indeterminate form when the value of denominator $\sin \left(\omega_{0} d / 2 v\right)$ approaches to zero, or $\bar{S}_{\text {res }}=d /\left.(2 j L)\right|_{j=1,2,3 \ldots}$. This is exactly the resonance condition for a simply supported compound beam subjected to a series of moving loads with identical intervals. Correspondingly, when $j$ is set to be 1 , the resonance response is called the main resonant peaks and the resonant speed is denoted as $v_{\text {res }}$ in the present study. By L'Hospital rule, the dynamic factor $Q_{2}\left(v_{\text {res }}, t\right)$ for resonance in Equation (17d) can be manipulated to yield $[22,26]$ :

$$
\begin{aligned}
Q_{2}\left(v_{r e s}, t\right)= & 2(N-1)\left[-\bar{S}_{r e s} \frac{\cos \left(\omega_{0} L / 2 v_{r e s}\right)}{1-\bar{S}_{r e s}^{2}}\right] \\
& \times \sin \omega_{0}\left(t-\frac{L}{2 v_{r e s}}\right)
\end{aligned}
$$

where the subscript res means resonance. The preceding equation indicates that larger response will be induced on the main beam of the compound beam when there are more loads moving at resonant speed vres, as implied by $(N-1)$ in Equation (19). And it is concluded that due to the repetitive nature of train-loads when the excitation circular-frequency $v / d$ caused by the running speed of the moving train loads is close to the fundamental circular-frequency $\omega_{o} / 2 \pi$ of the compound beam, the resonant speed is equal to $v_{\text {res }}=\omega_{0} d / 2 \pi$. It is worthy to mention that the resonant condition for railway bridges subjected to the train loads moving at high speeds has also been presented in related papers $[7,8,10,11]$.

\section{INCREAMENTAL-ITERATIVE METHOD}

Since the expression of the reaction force $F_{y}$, which applies to the main beam, is highly nonlinear in terms of the midpoint displacement $\Delta_{0}$ in Equation (8) and the summation term $\sum_{j=1}^{\infty}\left(j \pi q_{j} / L\right)^{2}$ in Equation (14d), an incremental-iterative method for computing the nonlinear dynamic response of Equation (13) is proposed in this section.

Substituting the expression of $F_{y}$ in terms of $\Delta_{0}$ in Equation (8) into Equation (13) yields

$$
\begin{aligned}
& \ddot{q}_{n}+2 \xi_{n} \omega_{n} \dot{q}_{n}+\omega_{n}^{2} q_{n}+\bar{F}_{n}(t)=F_{n}(t) \\
& \bar{F}_{n}(t)=\frac{2}{m L}\left[M \ddot{\Delta}_{0}+c_{t} \dot{\Delta}_{0}+\frac{E_{t} A_{t}}{l_{t, 0}^{3}} \Delta_{0}\left(h+\Delta_{0}\right)\left(2 h+\Delta_{0}\right)\right] \\
& \times \sin \frac{n \pi}{2}+\frac{E A}{m}\left(\frac{n \pi}{L}\right)^{2}\left[\sum_{j=1}^{\infty}\left(\frac{j \pi}{L} q_{j}\right)^{2}\right] q_{n} \\
& \Delta_{0}=\sum_{n=1}^{\infty} q_{n}(t) \sin \frac{n \pi}{2}
\end{aligned}
$$

Equation (20) shows that it is a set of highly non-linearcoupled differential equations in terms of the generalized displacements $q_{n}$ and the midpoint displacement $\Delta_{0}$ of the main beam. The solution of such simultaneously nonlinear equations can be performed by the Newmark$\beta$ finite difference scheme and incremental-iterative method. The Newmark- $\beta$ method is employed to discretize the differential equation of motion into an equivalent incremental equation, and the incrementaliterative procedure is divided into three phases: predictor, corrector and equilibrium checking, for solving the nonlinear equations. The predictor phase is concerned with the solution of structural response increments based on structural equation, as it is well known for iterative schemes, the tangent stiffness matrix used in the predictor phase need not be exact $[6,23]$. Therefore, the same (approximate) tangent stiffness matrix is used by the modified Newton-Raphson method [17] for all the iterative steps performed at each incremental step in the present study. As for the corrector phase, the accurately internal force recovery from the displacement increments calculated by the predictor phase is mainly concerned. Finally, the equilibrium phase deals with the force equilibrium checking between the internal forces of structures and the external exciting forces. The force equilibrium phase determines the unbalanced forces for the nonlinear vibrating structures by comparing the internal forces with the external loads. Once the unbalanced forces do not satisfy the non-negligible level, iterations for structural equilibrium involving the predictor and corrector phases are repeated until the tolerance of convergence is reached. Therefore, the solution of the nonlinear coupled equations given in Equation (20) is usually attempted by 
the incremental-iterative method, which is characterized by three major phases described below.

\section{Predictor phase}

Consider the differential equation of Equation (20) and let $q_{n, t+\Delta t}$ denote the total generalized coordinate at the time $t+\Delta t$ and $\Delta q_{n}$ the increments from time $t$ to $t+$ $\Delta t$, that is, $q_{n, t+\Delta t}=q_{n, t}+\Delta q_{n}$. Equation (20) can be rewritten as

$$
\begin{aligned}
\ddot{q}_{n, t+\Delta t}+ & 2 \xi_{n} \omega_{n} \dot{q}_{n, t+\Delta t}+\omega_{n}^{2}\left(q_{n, t}+\Delta q_{n}\right)+\bar{F}_{n}(t+\Delta t) \\
= & F_{n}(t+\Delta t) \\
\bar{F}_{n}(t+\Delta t)= & \frac{2}{m L}\left[\frac{E_{t} A_{t}}{l_{t, 0}^{3}} \Delta_{0, t+\Delta t}\left(h+\Delta_{0, t+\Delta t}\right)\left(2 h+\Delta_{0, t+\Delta t}\right)\right. \\
& \left.+M \ddot{\Delta}_{0, t+\Delta t}+c_{t} \dot{\Delta}_{0, t+\Delta t}\right] \sin \frac{n \pi}{2} \\
& +\frac{E A}{m}\left(\frac{n \pi}{L}\right)^{2}\left[\sum_{j=1}^{\infty}\left(\frac{j \pi}{L} q_{j, t+\Delta t}\right)^{2}\right] q_{n, t+\Delta t} \\
\Delta_{0, t+\Delta t}= & \sum_{n=1}^{\infty} q_{n, t+\Delta t} \sin \frac{n \pi}{2}
\end{aligned}
$$

With the previous description that the tangent stiffness matrix used in the predictor phase need not be exact during the iterative stage, as shown in Equation (21a), let us bring the force term $\bar{F}_{n}(t+\Delta t)$ to the right side of the equal sign and then use the Newmark finite difference scheme with constant average acceleration [12] to discretize the differential equations at predictor phase, the equivalent incremental equations at the time $t+\Delta t$ are described by the following form:

$$
\begin{aligned}
\left(a_{0}+a_{1} \frac{c}{m}+\right. & \left.\omega_{n}^{2}\right) \Delta q_{n}=\Delta F_{n}(t+\Delta t) \\
\Delta F_{n}(t+\Delta t) & =F_{n}(t+\Delta t)-\bar{F}_{n}(t+\Delta t)-\left[\omega_{n}^{2} q_{n, t}\right. \\
& +\left(a_{2} \dot{q}_{n, t}+a_{3} \ddot{q}_{n, t}\right) \\
& \left.+2 \xi_{n} \omega_{n}\left(a_{4} \dot{q}_{n, t}+a_{5} \ddot{q}_{n, t}\right)\right]
\end{aligned}
$$

Here, the coefficients ai referred from the reference [12] are expressed as

$$
\begin{aligned}
& a_{0}=\frac{1}{\beta \cdot \Delta t^{2}} ; a_{1}=\frac{\gamma}{\beta \cdot \Delta t} ; a_{2}=\frac{1}{\beta \cdot \Delta t} ; a_{3}=\frac{1}{2 \beta}-1 ; \\
& a_{4}=\frac{\gamma}{\beta}-1 ; a_{5}=\frac{\Delta t}{2}\left(\frac{\gamma}{\beta}-2\right) ; a_{6}=(1-\gamma) \Delta t ;
\end{aligned}
$$

$$
a_{7}=\gamma \cdot \Delta t
$$

where the parameters $\beta=0.25$ and $\gamma=0.5$. It is noted that the effective force increment $\Delta F_{n}(t+\Delta t)$ in Equation (22b) is not exact due to the approximately force term $\bar{F}_{n}(t+\Delta t)$ in association with the nonlinear expressions of the generalized displacements $q_{n, t}+\Delta t$ and the midpoint displacement $\Delta_{0, t+\Delta t}$, as shown in Equation (21b). Therefore, an iterative procedure of corrector phase for solving the nonlinear equations is described in the following section.

\section{Corrector phase}

For the purpose of carrying out the incrementaliterative procedure, the equivalent stiffness equation of the incremental generalized displacements $\Delta q_{n}$ at the $i$ th iteration of time $t+\Delta t$ in Equation (22) is expressed as $[16,17,23]$

$$
\begin{aligned}
& \left(a_{0}+a_{1} \frac{c}{m}+\omega_{n}^{2}\right) \Delta q_{n}=\Delta F_{n}^{i-1} \\
& \Delta F_{n}^{i-1}=F_{n}(t+\Delta t)-F_{n, i n t}^{i-1}(t+\Delta t) \\
& F_{n, i n t}^{i-1}(t+\Delta t)=\omega_{n}^{2} q_{n, t+\Delta t}^{i-1}+\left(a_{2} \dot{q}_{n, t+\Delta t}^{i-1}+a_{3} \ddot{q}_{n, t+\Delta t}^{i-1}\right) \\
& +2 \xi_{n} \omega_{n}\left(a_{4} q_{n, t+\Delta t}^{i-1}+a_{5} \ddot{q}_{n, t+\Delta t}^{i-1}\right)+\bar{F}_{n}^{i-1}(t+\Delta t)
\end{aligned}
$$

$(23 a-c)$

Here the right superscript $i$ indicates the current number of iterations. The term $\Delta F_{n}^{i-1}$ in Equation (23b) represents the load increment and $F_{n \text {,int }}^{i-1}(t+\Delta t)$ the internal force obtained from Equation (23c). Accordingly, the responses of displacement, acceleration and velocity of the generalized system for the ith current iterative step at the time $t+\Delta t$ are respectively given as [21]

$$
\begin{aligned}
& q_{n, t+\Delta t}^{i}=q_{n, t+\Delta t}^{i-1}+\Delta q_{n} \\
& \ddot{q}_{n, t+\Delta t}^{i}=a_{0} \Delta q_{n}^{i}-a_{2} \dot{q}_{n, t+\Delta t}^{i-1}-a_{3} \ddot{q}_{n, t+\Delta t}^{i-1} \\
& \dot{q}_{n, t+\Delta t}^{i}=\dot{q}_{n, t+\Delta t}^{i}+a_{6} \ddot{q}_{n, t+\Delta t}^{i-1}+a_{7} \ddot{q}_{n, t+\Delta t}^{i}
\end{aligned}
$$

Once the responses $\left(q_{n, t+\Delta t}^{i}, \dot{q}_{n, t+\Delta t}^{i}, \ddot{q}_{n, t+\Delta t}^{i}\right)$ of the generalized system at the ith iteration of the time $t+\Delta t$ are made available, the internal force $F_{n, i n t}^{i-1}(t-\Delta t)$ of the generalized system, as given in Equation (23c), can be computed. Substituting Equation (24) into Equation $(23 \mathrm{c})$, one can update the new internal force term $F_{n, \text { int }}^{i}(t+\Delta t)$. Next, let $F_{n, \text { int }}^{i-1}(t+\Delta t)=F_{n \text {,int }}^{i}(t+\Delta t)$ and find the unbalanced force $\Delta F_{n}^{i-1}$ shown in Equation (23b), the equilibrium checking phase can be carried out. 


\section{Equilibrium checking phase}

In this phase, updating the total responses of generalized coordinates shown in Equation (24), calculating the internal forces from Equation (23c), and comparing the internal forces with the external loads, one can compute the unbalanced forces $\Delta F_{n}^{i-1}$ shown in Equation (23b). Whenever the unbalanced forces are larger than a preset tolerance, iterations for equilibrium of the structure involving the two phases, i.e., predictor and corrector, are repeated until the condition of force equilibrium is convergent to an allowable tolerance. Then, the moving load-induced response of the compound beam can be calculated by the conventional procedure of direct integration method [1].

\section{DYNAMIC RESPONSE FACTOR AND SPEED PARAMETER}

In this study, dynamic response factor DRF is used to account for the amplification effect of a simply supported beam installed with a two-members truss system to moving train-loads. It is defined as follows

$$
D R F=\frac{R_{d}(x)}{R_{s}(x)}
$$

where $R_{d}(x)=$ the maximum dynamic response of the simple beams with two-member trusses calculated at position $x$ and $R_{x}(x)=$ the maximum static response of a simply supported beam. On the other hand, the speed parameter $S$ is defined as the ratio of the excitation frequency of moving loads, i.e., $\pi v / L$, with $v$ denoting the vehicle speed and $L$ the span length, to the fundamental frequency $\omega_{1}$ of the simply supported beam, i.e., $S=\pi v / \omega_{1} L$. Therefore, in this study, the relationship between $D R F$ and $S$ is named as $D R F-S$ plot.

\section{ILLUSTRATIVE EXAMPLE}

For the purpose of illustration, let us consider a simply supported beam, which is made of steel, the properties of the simple beam are listed in Table 1. A

Table 1. Properties of the simply supported beam

\begin{tabular}{cccccc}
\hline$L(\mathrm{~m})$ & $m(\mathrm{t} / \mathrm{m})$ & $E I\left(\mathrm{kN}-\mathrm{m}^{2}\right)$ & $\xi_{n}$ & $E A(\mathrm{kN})$ & $\omega_{1}(\mathrm{rad} / \mathrm{s})$ \\
\hline 24 & 11 & $2.5 \times 10^{7}$ & $1.5 \%$ & $2 \times 10^{7}$ & 25.83 \\
\hline
\end{tabular}

Table 2. Properties of the truss member and rigid bar

\begin{tabular}{cccc}
\hline$E_{t} A_{t}(\mathrm{kN})$ & $m_{t}(\mathrm{t} / \mathrm{m})$ & $m_{r}(\mathrm{t} / \mathrm{m})$ & $c_{t}(\mathrm{kN}-\mathrm{s} / \mathrm{m})$ \\
\hline $2 \times 10^{6}$ & 0.1 & 0.2 & 0.0 \\
\hline
\end{tabular}

damping ratio $\xi_{n}=1.5 \%$ is assumed for the steel beam in this study [5, 6]. Moreover, an undamped twomember steel truss is considered and installed in the main beam through the connection of a set of rigid bars, as shown in Figure 1. The properties of the two-member truss system are shown in Table 2 . The traveling load model is assumed to have $N=20$ and constant interval $d=18 \mathrm{~m}$, and the weight of lumped moving load is set to be $P=270 \mathrm{kN}$.

\section{Resonance of simple beams with two-member trusses}

This example is to illustrate the phenomenon of resonance of a simple beam subjected to moving loads and the effect of strengthening by installing a twomember truss system in the main beam. Using the properties shown in Tables 1 and 2, and considering the aspect ratio $h / L=0.15$ for the compound beam of which the fundamental frequency $\omega_{0}$ is equal to $1.14 \omega_{1}$ computed from Equation (17e), the $D R F-S$ plots of midpoint displacement response for the simple beam and the compound beam are drawn in Figure 4, respectively. The numerical results indicate that the main resonant response of the simple beam traveled by the moving loads can be found at the resonant speed parameter $S_{\text {res, } 1}=0.375\left(v_{\text {res }}=292 \mathrm{~km} / \mathrm{h}\right)$, which agrees exactly with the resonant condition mentioned previously, that is, $S_{r e s, 1}=d / 2 L=18 /(2 * 24)=0.375$. On the other hand, another resonant peak of the compound beam with the aspect ratio $h / L=0.15$ is also found at the resonant speed parameter $S_{\text {res, } 2}=0.426$, which is equal to $S_{\text {res }, 1}$ $\times \omega_{0} / \omega_{1}=0.426\left(v_{\text {res }}=333 \mathrm{~km} / \mathrm{h}\right)$. As can be seen from the time history response resonance plotted in Figure 5, it shows that the midpoint displacement response of the simple beam is larger than that of the compound beam. Here, $\Delta_{s m p}$ is denoted the maximum midpoint static deflection of the simply supported beam under the action of a concentrated force applying to the midpoint

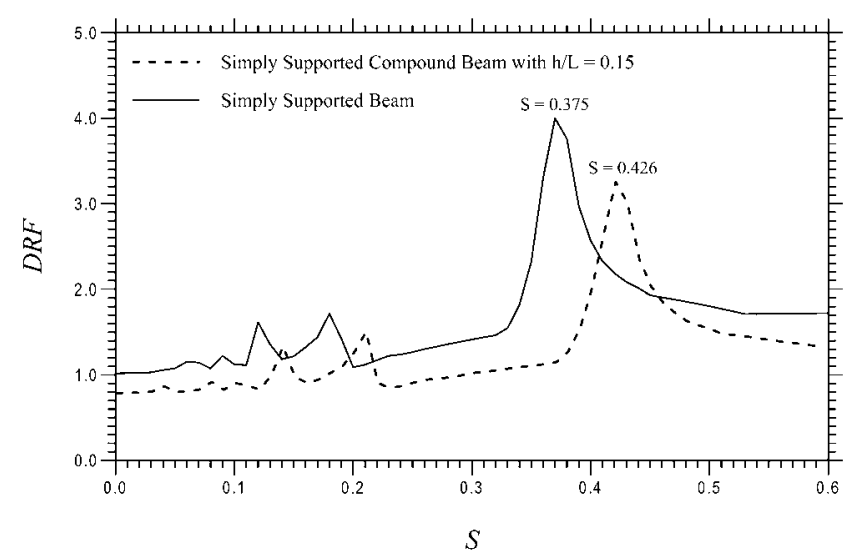

Fig. 4. Dynamic response factor $D R F$ vs. speed parameter $S$. 
of the beam. This is mainly due to the strengthening effect of installing a two-member truss system in the main beam.

On the other hand, to comprehend the influence of nonlinear effect on the behavior of truss members, the dynamic responses of internal force $f_{x}$ in the truss members attached to the main beam with an aspect ratio $h / L$ $=0.15$ are plotted in Figure 6 . The numerical results indicate that the response with the consideration of nonlinear vibration is slightly larger than that with only considering linear effect. According to the present study, although the nonlinear effect of truss members is not so significant to the moving-load induced response of the compound beam, it is necessary to take into account the nonlinear vibration for accurately computing the response of truss member in a compound beam structure subjected to moving-train loads in this study. Moreover, to investigate the train-induced response of a simple beam installed with different two-member trusses, a further investigation of the effect of strengthening by aspect ratio $(h / L)$ will be demonstrated in the

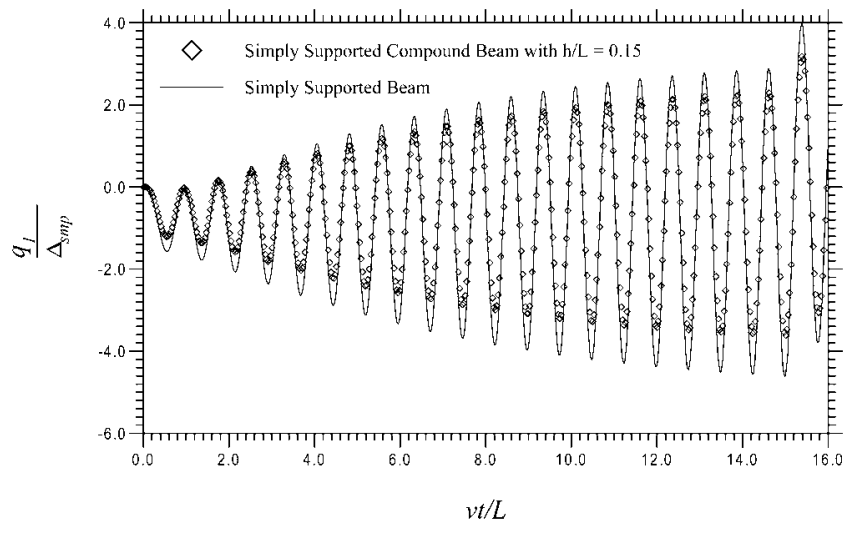

Fig. 5. Time history responses at resonant speeds.

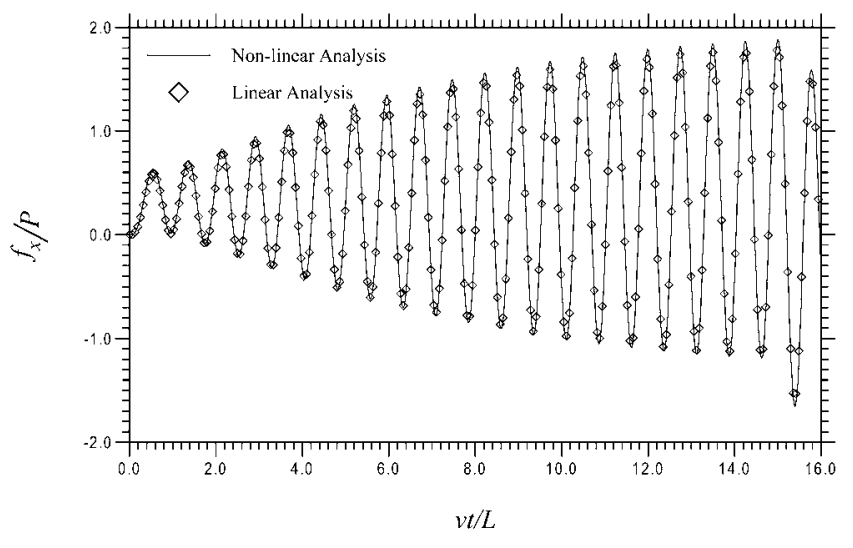

Fig. 6. Time history responses of internal force of truss member at resonant speed. next example.

\section{Effect of strengthening of two-member trusses on simple beams}

To illustrate the dynamic strengthening effect of the two-member truss on a simply supported beam, different aspect ratios $(h / L)$ ranging from 0 to 0.5 are considered in this study. Based on the incrementaliterative procedure, the numerical results are given in Figure 7, which the dynamic response factor $D R F$ computed for the midpoint displacement of the main beam has been plotted against the speed parameter $S$ and the aspect ratio $h / L$. As can be seen at the main resonant speed parameter, the larger the aspect ratio of the compound beam, the smaller the displacement response of the main beam will be. Meanwhile, there is a trend of the smaller values of $D R F$ as increasing the aspect ratio. This means that increasing the aspect ratio $(h / L)$ may give a more dynamic strengthening effect to the compound beam.

\section{CONCLUDING REMARKS}

This study presents the dynamic response of a simple beam with a two-member truss system to moving-train loads. Based on the nonlinear theory of a symmetric two-member truss subjected to a vertical tip load and the incremental-iterative solution procedures for solving a nonlinear dynamic system, the dynamic

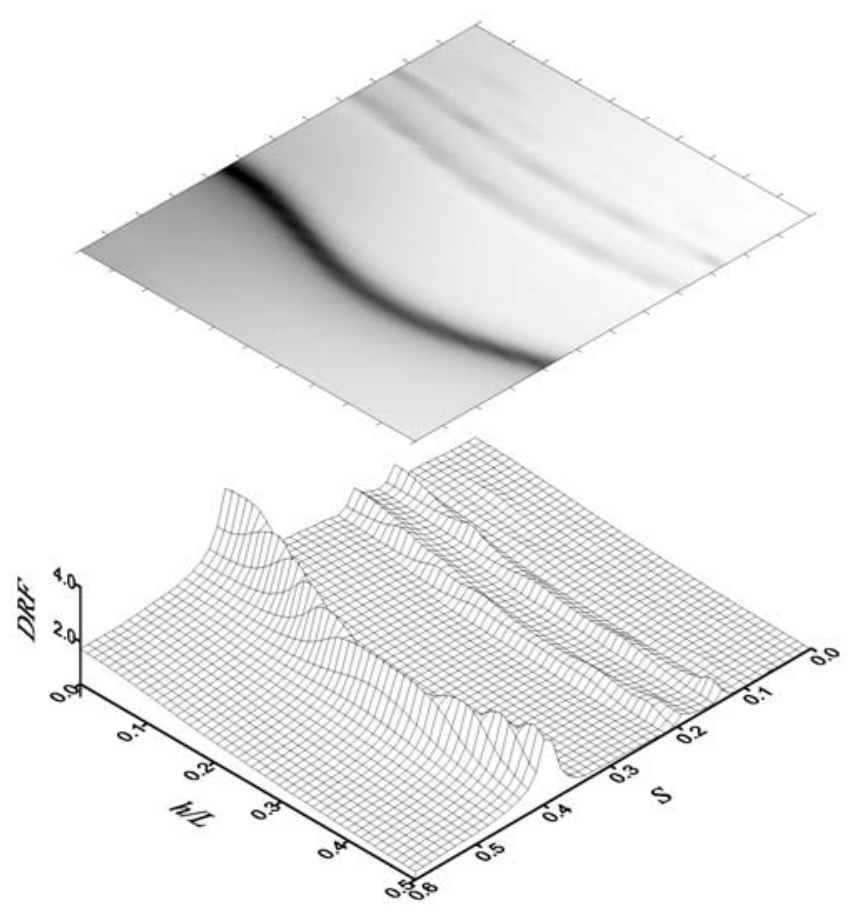

Fig. 7. Effect of aspect ratio $h / L$ on $D R F-S$ plot. 
response of a compound beam under moving load excitations can be computed. Although the nonlinear effect of vibration is not so obvious to the response of the beam, but the numerical examples demonstrated that increasing the aspect ratio $h / L$ could effectively reduce the main resonant response of the compound beam due to moving loads.

\section{ACKNOWLEDGEMENT}

The research reported herein has been sponsored in part by the National Science Council of the Republic of China through grants No. NSC93-2211-E-032-010. Such a financial aid is gratefully acknowledged. Parts of the illustrative examples of this paper is modified from the paper entitled "Non-linear Vibrations of Simple Truss-Beams to Moving Loads," presented at the Book of Abstract, 5th Euromech Solid Mechanics Conference ESMC003 [25], held during Aug., 2003 at Thessaloniki, Greece.

\begin{tabular}{|c|c|}
\hline & OMENCLATURE \\
\hline$a_{i}$ & $\begin{array}{l}\text { coefficients of Newmark finite dif- } \\
\text { ference scheme }\end{array}$ \\
\hline$c$ & damping coefficient of the beam \\
\hline$c_{t}$ & $\begin{array}{l}\text { damping coefficient of the truss } \\
\text { member, }\end{array}$ \\
\hline$e_{x}$ & the axial strain \\
\hline DRF & dynamic response factor \\
\hline$E A$ & axial rigidity \\
\hline$E_{t} A_{t}$ & axial rigidity of truss member \\
\hline EI & flexural rigidity of the beam \\
\hline$f_{x}$ & $\begin{array}{l}\text { the vertical component of internal } \\
\text { force in the truss mmeber }\end{array}$ \\
\hline$F_{n}(t)$ & generalized forcing function \\
\hline $\bar{F}_{n}(t)$ & $\begin{array}{l}\text { internal force defined in Equation } \\
(20 \mathrm{~b})\end{array}$ \\
\hline$F_{n, \text { int }}^{i-1}(t+\Delta t)$ & $\begin{array}{l}\text { internal force defined in Equation } \\
(23 \mathrm{c})\end{array}$ \\
\hline$F_{y}$ & reaction force \\
\hline$h$ & the height of two-member truss \\
\hline$H(t)$ & unit step function \\
\hline$l_{t, 0}$ & $\begin{array}{l}\text { undeformed length of the truss mem- } \\
\text { ber }\end{array}$ \\
\hline$l_{t, 2}$ & $\begin{array}{l}\text { deformed length of the truss mem- } \\
\text { ber }\end{array}$ \\
\hline$L$ & span length \\
\hline$m$ & mass per unit length of the beam \\
\hline$m_{r}$ & $\begin{array}{l}\text { the mass per unit length of the rigid } \\
\text { bar }\end{array}$ \\
\hline$m_{t}$ & $\begin{array}{l}\text { the mass per unit length of the truss } \\
\text { member }\end{array}$ \\
\hline$N$ & total number of moving loads \\
\hline
\end{tabular}

\begin{tabular}{|c|c|}
\hline$p(x, t)$ & load function \\
\hline$q_{n}(t)$ & the generalized coordinate \\
\hline$Q_{1}(v, t), Q_{2}(v, t)$ & dynamic factor \\
\hline$R_{d}(x)$ & the maximum dynamic response \\
\hline$R_{s}(x)$ & the maximum static response \\
\hline$R_{y}$ & $\begin{array}{l}\text { the internal force acting on the tip } \\
\text { node }\end{array}$ \\
\hline$S$ & speed parameter $=\pi v / \omega_{1} L$ \\
\hline $\bar{S}_{\text {res }}$ & speed parameter $=d /\left.2 j L\right|_{j=1,2,3 \ldots}$ \\
\hline$t_{k}=(k-1) d / v$ & $\begin{array}{l}\text { arriving time of the kth load on the } \\
\text { beam }\end{array}$ \\
\hline$u(x, t)$ & axial displacement of the beam \\
\hline$v_{\text {res }}$ & resonant speed \\
\hline$w(x, t)$ & transverse deflection of the beam \\
\hline$x$ & beam axis \\
\hline$\alpha$ & $\tan ^{-1}(2 h / L)$ \\
\hline$\delta$ & Dirac's delta function \\
\hline$\Delta_{0}$ & midpoint deflection of the beam \\
\hline & the maximum static deflection \\
\hline$\Delta F_{n}^{l-1}$ & $\begin{array}{l}\text { the load increment at the ith itera- } \\
\text { tion step }\end{array}$ \\
\hline$\xi_{n}$ & damping ratio \\
\hline$\omega_{0}$ & the fundamental frequency \\
\hline$\omega_{n}$ & the nth frequency of a simple beam \\
\hline
\end{tabular}

\section{REFERENCES}

1. Bathe, K.J., Finite Element Procedures in Engineering Analysis, Prentice-Hall, New Jersey (1982).

2. Chakraborty, G., Mallik, A.K., and Hatwal, H., "NonLinear Vibration of a Travelling Beam," Intl. J. Nonlinear Mech., Vol. 34, No. 2, pp. 655-670 (1999).

3. Fryba, L., Vibration of Solids and Structures Under Moving Loads, Noordhoff International Publishing, Groningen, The Netherlands (1972).

4. Fryba, L., Dynamics of Railway Bridges, Thomas Telford, London (1996).

5. Fryba, L., "Intensive Vibration of Bridges due to High Speed Trains," Proceedings of Computers in Railways VIII, Allan et al., (Eds.), WIT Press, Southampton, U.K. (2002).

6. Fryba, L., Fischer, C., and Yau, J.D. "Stress Ranges in Bridges Under High Speed Trains," Proceedings of the $9^{\text {th }}$ Intl. Conference on Civil and Structural Engineering Computing, Topping, B.H.V. (Ed.), CIVIL-COM Press, (2003).

7. Fryba, L. and Naprstek, J., “Appearance of Resonance Vibration on Railway Bridges," Proceedings of Advances in Civil and Structural Engineering Computing, Topping, B.H.V. (Ed.), Civil-Comp Press, Edingburg, Scotland, pp. 377-382 (1998).

8. Klasztorny, M., "Vertical Vibrations of a Multi-Span Beam Bridge Under a Train Moving at High Speeds," Proceedings of the $4^{\text {th }}$ European Conference on Struc- 
tural Dynamics-EURODYN'99, Fryba, L. and Naprstek, J. (Eds.), Balkema, Rotterdam, pp. 651-656 (1999).

9. Kwon, H.C., Kim, M.C., and Lee, I.W., "Vibration Control of Bridges Under Moving Loads," Comput. Struct., Vol. 66, No. 4, pp. 473-480 (1998).

10. Liberatore, D., "Dynamic Interaction Between Periodic Train and Bridge at Resonance," Proceedings of the $4^{\text {th }}$ European Conference on Structural DynamicsEURODYN'99, Fryba, L. and Naprstek, J. (Eds.), Balkema, Rotterdam, pp. 693-698 (1999).

11. Museros, P., Vivero, G., and Alarcon, E., "Moving Loads on Railway Bridges: The Spanish Code Approach," Proceedings of the $4^{\text {th }}$ European Conference on Structural Dynamics-EURODYN'99, Fryba, L. and Naprstek, J. (Eds.), Balkema, Rotterdam, pp. 675-680 (1999).

12. Newmark, N.M., "A Method of Computation for Structural Dynamics," J. Eng. Mech. Div., ASCE, Vol. 85, No. 1, pp. 67-94 (1959).

13. Öz, H.R., Pakdemirli, M., and Boyaci, H., "Non-Linear Vibrations and Stability of an Axially Moving Beam with Time-Dependent Velocity," Intl. J. Nonlinear Mech., Vol. 36, No. 1, pp. 107-115 (2001).

14. Villaggio, P., Mathematical Models for Elastic Structures, Cambridge University Press, Cambridge, UK (1997).

15. Wang, J.F., Lin, C.C., and Chen, B.L., "Vibration Suppression for High-Speed Railway Bridges Using Tuned Mass Dampers," Intl. J. Solids Struct., Vol. 40, No. 2, pp. 465-491 (2003).

16. Yang, Y.B. and Chen, Y.C. "Design of Sliding-Type Base Isolators by the Concept of Equivalent Damping," Struct. Eng. Mech., Vol. 8, No. 3, pp. 299-310 (1999).

17. Yang, Y.B. and Kuo, S.R. Theory and Analysis of Nonlinear Framed Structures, Prentice-Hall, Singapore (1994).
18. Yang, Y.B., Liao, S.S., and Lin, B.H., "Impact Formulas for Vehicles Moving over Simple and Continuous Beams," J. Struct. Eng., ASCE, Vol. 121, No. 11, pp. 1644-1650 (1995).

19. Yang, Y.B., Lin, C.L., Yau, J.D., and Chang, D.W., "Mechanism of Resonance and Cancellation for TrainInduced Vibrations on Bridges with Elastic Bearings," J. Sound Vib., Vol. 269, No. 1-2, pp. 345-360 (2004).

20. Yang, Y.B. and Wu, Y.S. "Chaotic Behaviours of a TwoMember Truss," J. Vib. Control, Vol. 3, No. 1, pp. 103118 (1997).

21. Yang, Y.B. and Yau, J.D., "Vehicle-Bridge Interaction Element for Dynamic Analysis," J. Struct. Eng., ASCE, Vol. 123, No. 11, pp. 1512-1518 (1997).

22. Yang, Y.B., Yau, J.D., and Hsu, L.C., "Vibration of Simple Beams Due to Trains Moving at High Speeds," Eng. Struct., Vol. 19, No. 11, pp. 936-944 (1997).

23. Yang, Y.B., Yau, J.D., and Leu, L.J., "Recent Development on Nonlinear and Postbuckling Analysis of Framed Structures," Appl. Mech. Rew., Vol. 56, No. 4, pp. 431449 (2003).

24. Yau, J.D., "Resonance of Continuous Bridges Due to High Speed Trains," J. Mar. Sci. Technol., Vol. 9, No. 1, pp. 14-20 (2001).

25. Yau, J.D., "Non-linear Vibrations of Simple TrussBeams to Moving Loads," Book of Abstract, 5th EuroMech Solid Mechanics Conference ESMC003, Thessaloniki, Greece (2003).

26. Yau, J.D., Wu, Y.S., and Yang, Y.B., "Impact Response of Bridges with Elastic Bearings to Moving Loads," J. Sound Vib., Vol. 248, No. 1, pp. 9-30 (2001).

27. Yau, J.D., and Yang, Y.B., "Vibration Reduction for Cable-Stayed Bridges Traveled by High Speed Trains," Finite Element Anal. Des., Vol. 40, No. 3, pp. 341-359 (2004). 